\title{
The Research of Relationship Between BMI Classification and Physical Function of Male College Students
}

\author{
Gaohua Li \\ The college of Henan university of agriculture,Zhengzhou,He Nan province,china. \\ gaohuali1013@163.com.
}

\begin{abstract}
This paper explored the effects of body mass index classification and college students' physical function index in a study of the 1364 male students Henan university of agriculture subjects were divided into 4 groups. The result showed that the variation on physical fitness indicators among the different body composition differs from one another ,the cardiovascular function, respiratory function, arm strength and lower limb explosive power of nomal weight group is superior to the low weight(except for respirtory function),over weight and obese group(except for arm strength),low weight(except for respirtory function),Over-weight and obesity all have negative effects on students'physical function.
\end{abstract}

Keywords-BMI; male college students; physical function

\section{INTRODUCTION}

Statistical analysis of the 2010 National Student Fitness and health survey and data has come to an end. Investigation showed a decreasing trend that a number of indicators of the health of college students in China. Students 19 to 22 age group, the explosive force, strength, endurance levels decline further but flexibility index[1].

Body mass index is a reflection of the relationship between adult body weight and height, be used to judge the body fat levels are not influenced by gender[2]. The present research about BMI is mainly focused on the relationship between obesity and diseases, but the relationship between BMI and physical health of male college students study, the results of studies are difference[2,3].

The purpose of this study was to explore the relationship between the male students BMI and "Student Physique Healthy Standard" contains the physical fitness of college students, distribution characteristics analysis of male college students BMI, revealing the influence of abnormal BMI on College Students' physical health of college students health standard, which causes decreased, and use scientific sports to exercise as a means guidance for college students.

\section{METHODS AND SUBJECTS}

\section{A. Subjects}

The subjects used in this study were the Henan Agricultural University level 2011 male university
students.Testing according to the "Student Physique Healthy Standard", the test data are: height, weight, vital capacity, body mass index, grip strength, standing long jump, $1000 \mathrm{~m}$. The effective data and the use of 1364. Age distribution was $19 \sim 22$ years old. Participants from the different majors, including fourteen provinces and autonomous regions nationwide student.

\section{B. Methods}

The definition and the calculation method of BMI.According to the WHO recommended adult BMI value, the research object of this paper is divided into four groups according to BMI: 1) low body weight BMI $<18.5$, normal weight group BMI in 18.5-23.9 BMI 24-27.9, overweight, obesity group BMI $\geq 28$ [4].

Vital capacity weight index, grip and body mass index formula:Vital capacity weight index $=$ vital capacity $(\mathrm{ml}) /$ body weight $(\mathrm{kg})$; grip BMI $=($ grip strength $(\mathrm{kg}) /$ body weight $(\mathrm{kg})) \times 100$.

\section{Experimental Protocol}

Physical health test.According to the "Student Physique Healthy Standard " to test. The first measurement of the height, weight, vital capacity, and then test the grip strength, standing long jump, $1000 \mathrm{~m}$.

\section{Statistical Analysis}

The analysised used of SPSS17.0 to to analyze the data:The frequency table method, variance analysis, chi-square test, $t$ test and other statistical methods.

\section{RESULTS}

\section{A. Distribution characteristics of body mass index of College Students.}

By the frequency analysis, BMI distribution curve of college students see (Figure 1) peak at about 21, indicates that the BMI is relatively concentrated, most of the BMI distribution in the normal range, but there are a certain number of low birth weight and overweight or obese students. 


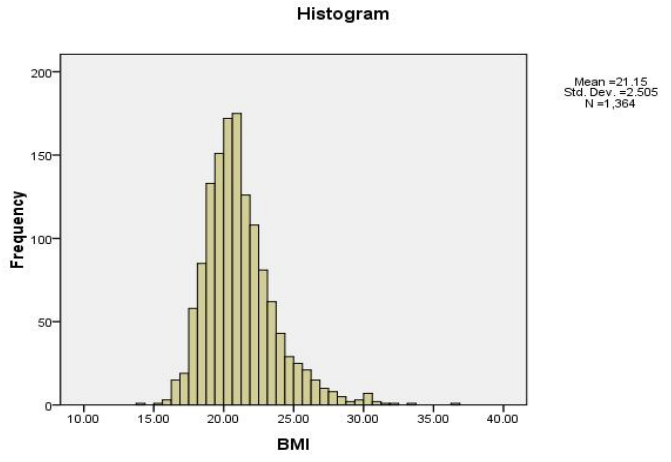

Figure1 MaLe Students BMI Distribution Histogram

Table1 Frequency TABle BMi Distribution of Male College STUDENTS

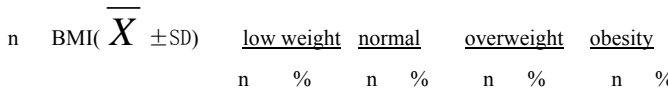

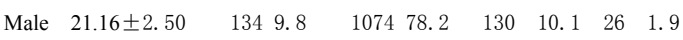

Table 1 shows that the distribution of BMI value, the male students are normal basically, The number of BMI18.5-24 is 1074 , accounting for $78.2 \%$ of the total, overweight and obesity accounted for $6.2 \%$.

\section{B. Relationship between BMI index and health index(table2)}

TABLE 2 THE RELATIONSHIP BETWEen STUDENTS BMI VALUE AND Physical Health INDICES.

\begin{tabular}{|c|c|c|c|c|c|c|}
\hline Vital capacity & $\begin{array}{l}\mathrm{y} 66.52 \\
\end{array}$ & 73.02 & 67.25 & 57.26 & 499.17 & $52.81<0.001$ \\
\hline weight index & $x \pm 11.13$ & \pm 10.94 * & \pm 10.38 & \pm 9.07 * & \pm 7.91 * & \\
\hline $1000 \mathrm{~m}$ & 215.49 & 220.01 & 212.36 & 228.34 & 257.22 & $5.12<0.05$ \\
\hline & \pm 21.42 & \pm 23.40 & \pm 17.93 & \pm 24.75 & \pm 44.10 * & \\
\hline grip strength & 45.5 & 40.88 & 45.67 & 48.38 & 50.69 & $4.18<0.05$ \\
\hline & \pm 7.32 & \pm 7.33 & \pm 6.94 & \pm 7.53 & $\pm 9.32 *$ & \\
\hline Standing & 2.37 & 2.34 & 2.38 & 2.29 & 2.19 & $6.20<0.05$ \\
\hline long jump & \pm 0.16 & \pm 0.18 & \pm 0.15 & \pm 0.16 & \pm 0.20 * & \\
\hline
\end{tabular}

In this study, Table 2 showed that the boys all physical health index (in addition to grip and normal weight group) were higher than that of low body weight (except the vital capacity of body mass index), overweight and obesity group, there is significant difference in normal somatic recombination; vital capacity index is lower than the low body weight, are higher than the other two groups; grip strength index with BMI index increased and showed increasing phenomenon, but there is no significant difference.

\section{DISCUSSION}

\section{A. The distribution of male college students BMI}

Most of the boys in the study of BMI distribution in the normal range, but there are still a number of low weight, overweight or obese students. College students are in the period of youth, the functions of the body in the best period, at this time, BMI abnormalities on physical health hazards are often difficult to express, therefore, the impact on College Students' health is often neglected.

The boys of BMI in this study was higher than that of other similar studies of girls, this result and many international research[5]. This may be related to the age of girls than boys more attention through the control diet, strengthening exercise, maintain body size. Physical education should strengthen the control of male weight exercise guidance, and strive to reduce overweight and obesity. Research has shown that, aerobic exercise can improve the abnormal metabolism of related with obesity [5]; and studies have shown that physical activity can improve the body composition of university students $[6,7]$.

\section{B. BMI and vital capacity weight index}

Vital capacity weight index is one of the physical health test composite index, an important index to evaluate human respiratory level[7]. Vital capacity weight index of boys in this study, for low body weight, were higher than that in the normal group, however, overweight and obesity group were lower than those of the normal group. The physiological ventilator overweight or obesity of college students can have a significant negative impact, its reason lies in: obesity caused by chest wall and abdominal fat thickness, so that the decline in lung capacity, vital capacity is reduced, affect normal lung ventilation function. Because the accumulation of fat, may also influence tracheal cilia activity, so it can not play the normal function, But the low weight's lung function are better than others.

\section{BMI and $1000 \mathrm{~m}$ performance in long distance run}

From table 2 showed, The effects of different BMI values on male endurance running, four groups of different body mass index of boys in endurance running there was a significant difference $(\mathrm{P}<0.05)$, normal group outperformed other abnormal BMI group. In the multiple comparison further average, found that being overweight, obese students endurance running performance is significantly worse than normal weight and low birth weight $(\mathrm{P}<0.05)$. This result and $\mathrm{Wu}$ Xinyu, Bo Yang study of male college students is consistent with the conclusion that [8].

\section{$D$. The relationship between BMI and the explosive force of lower limbs}

From table 2, overweight or obese boys standing long jump is significantly less than the BMI normal students. Standing long jump is the physical quality index to reflect the lower limb explosive force, with the increase of BMI beyond the normal range, the relative power of their limbs is decrease. 


\section{E. The relationship between BMI and grip.}

From table 2, effects of different BMI on boys grip BMI, four groups of different body shape of the students have a very significant difference in grip BMI. We can know from the $\mathrm{F}$ values of body mass index, grip strength is greatly affected by the different body mass index. From the average, BMI exceeds a certain range, the grip body mass index decreased significantly. In the multiple comparison further mean, can see the grip strength / weight overweight, obese boys was significantly worse than normal weight and low birth weight $(\mathrm{P}<0.01)$, low weight in normal weight, overweight and obesity group was lower than that, there are very significant differences.

Grip/BMI is isometric force measurement method, only the test of muscle in static state, in a certain angle joint muscle strength, and power function of isometric muscle strength of human body motion is not significant. The boys grip strength index and BMI index in this study has significant positive correlation. And from Bo Yang et al [3] of male college students of upper limb muscle relative strength enhanced with the increase of BMI value and consistent.

\section{CONCLUSION}

(1)From this study, the results showed male students BMI distribution basically normal, average at around 21. But in overweight and obesity subjects are proportional, must strengthen the aerobic exercise training.

(2) For overweight or obesity male college students, Physiological ventilator, aerobic capacity, lower limb explosive force have a significant negative impact on.
(3) Because this study only BMI and physical fitness of male college students, not related to the gender and body composition index, due to gender differences, and body fat percentage, each person's muscle content of different, so simply height and weight ratio may be misleading values of body fat were higher than. So suggest relevant research to comparative study of girls BMI and function index, and community composition test, with the two results of further study may be more reference value.

\section{REFERENCES}

[1] In 2010 the national student fitness and health survey results published by [J]. Chinese school physical education, 2011

[2] Hao Xu. BMI index of adults in Jiangsu province [J]. sports and science 2001, 22 (6):39- 43

[3] Bo Yang.BMI abnormal effect on physical health index of college students [J]. Chinese sports science and technology, 2005, 41 (2): 138-139.

[4] Yuehong Mo. Research on [J]. of different body mass index and physical fitness of college students of Nanjing Sport Institute were, 2011, 10 (2):115-117.

[5] Kimm SY,Glynn NW,Obarzanek E,et al .Relation between the changes in physical activity and body mass index during adolescence:a mult-icentre longitudinal study. Lancet, 2005 Jul23-29，366(9482)

[6] Lei Wei Yibing Yu et al. Study on the [J]. basketball player correlation between body composition and physical health. Journal of Guangzhou Sports University, 2009,29 (5): 108-111.

[7] Rongbao Luo. The change of [J]. body composition of female university in 8 weeks fitness running after the study of tissue engineering in China, 2008,12 (28): 5577-5580 [8] Wu Xinyu, Fu Xiaochun. Study on the relationship between body mass index and [J]. index of health condition of college students. Journal of Beijing Sport University, 2006,29 (8): 1087-1088. 